\title{
Nonvacuum initial states for cosmological perturbations of quantum-mechanical origin
}

\author{
Jérôme Martin* and Alain Riazuelo ${ }^{\dagger}$ \\ DARC, Observatoire de Paris, UPR 176 CNRS, 92195 Meudon Cedex, France \\ Mairi Sakellariadou ${ }^{\ddagger}$ \\ Theory Division, CERN, CH-1211 Geneva 23, Switzerland \\ and Centre for Theoretical Physics, University of Sussex, Brighton, Falmer BN1 9QH, United Kingdom
}

(Received 8 April 1999; published 28 March 2000)

\begin{abstract}
In the context of inflation, nonvacuum initial states for cosmological perturbations that possess a built in scale are studied. It is demonstrated that this assumption leads to a falsifiable class of models. The question of whether they lead to conflicts with the available observations is addressed. For this purpose, the power spectrum of the Bardeen potential operator is calculated and compared with the CMBR anisotropies measurements and the redshift surveys of galaxies and clusters of galaxies. Generic predictions of the model are a high first acoustic peak, the presence of a bump in the matter power spectrum and non-Gaussian statistics. The details are controlled by the number of quanta in the nonvacuum initial state. Comparisons with observations show that there exists a window for the free parameters such that good agreement between the data and theoretical predictions is possible. However, in the case where the initial state is a state with a fixed number of quanta, it is shown that this number cannot be greater than a few. On the other hand, if the initial state is a quantum superposition, then a larger class of initial states could account for the observations, even though the state cannot be too different from the vacuum. Planned missions such as the MAP and Planck satellites and the Sloan Survey will demonstrate whether the new class of models proposed here represents a viable alternative to the standard theory.

PACS number(s): 98.80.Cq, 98.70.Vc
\end{abstract}

\section{INTRODUCTION}

The observed large-scale structure in the Universe has been currently addressed, within the framework of gravitational instability, by two families of models: initial density perturbations can either be due to "freezing in" of quantum fluctuations of a scalar field (inflaton) during an inflationary era [1] or they may be seeded by a class of topological defects, naturally formed during a symmetry-breaking phase transition in the early Universe [2]. The recent bulk of observational and experimental data and, in particular, the cosmic microwave background anisotropy measurements, and the redshift surveys of the distribution of galaxies and clusters of galaxies, impose severe constraints on the two families of models, as well as on the variety of possible scenarios introduced within each family.

The simplest topological defect models of structure formation show conflicts with observational data. As first shown in Ref. [3], global topological defects models predict strongly suppressed acoustic peaks. While on large angular scales the predicted cosmic microwave background radiation (CMBR) spectrum is in good agreement with the Cosmic Background Explorer (COBE) measurements, on smaller angular scales the topological defect models cannot reproduce the data of the Saskatoon experiment. One can manufacture models [4] with structure formation being induced by scaling seeds, which lead to an angular power spectrum with the

\footnotetext{
*Email address: martin@edelweiss.obspm.fr

†Email address: Alain.Riazuelo@obspm.fr

‡Email address: Mairi.Sakellariadou@cern.ch
}

same characteristics (position and amplitude of acoustic peaks), as the one predicted by standard inflationary models. The open question is, though, whether such models are the outcome of a realistic theory. However, the most severe problem for topological defects models of structure formation is their predicted $[5,6]$ lack of large-scale power in the matter power spectrum, once normalized to COBE. Choosing scales of $100 h^{-1} \mathrm{Mpc}$, which are most probably unaffected by nonlinear gravitational evolution, standard topological defect models, once normalized to COBE, require a bias factor $\left(b_{100}\right)$ on scales of $100 h^{-1} \mathrm{Mpc}$ of $b_{100} \approx 5$, to reconcile the predictions for the density field fluctuations with the observed galaxy distribution. However, the latest theoretical and experimental studies favor a current value of $b_{100}$ close to unity.

In what follows, we shall place ourselves within the framework of cosmological perturbations of quantummechanical origin in the context of inflationary models. The inflationary paradigm was proposed in order to explain the shortcomings of the standard (big bang) cosmological model. In addition, it offers a scenario for the generation of the primordial density perturbations, which can lead to the formation of the observed large-scale structure.

The theory of cosmological perturbations of quantummechanical origin rests on two well-established theories. On the one hand, (linearized) general relativity allows a calculation of the evolution and the amplification of perturbations throughout the cosmic evolution; the mechanism at work being parametric amplification of the fluctuations due to the interaction of the perturbations with the background [7]. On the other hand, quantum field theory permits us to understand the origin of these perturbations. If the quantum fields 
are initially, i.e., at the beginning of inflation, placed in the vacuum state, then because of the Heisenberg principle, fluctuations are unavoidable. Moreover, the amplitude of these fluctuations is completely fixed.

Inflation, employing the theory of cosmological perturbations of quantum-mechanical origin, leads to definite predictions for the anisotropies of the CMBR, as well as for the power spectrum, which can be tested against experimental and observational data. In particular, simple models predicts a scale-invariant spectrum, with, provided the quantum fields are initially placed in the vacuum, Gaussian fluctuations.

Let us briefly discuss the observational data, namely, the CMBR anisotropies measurements and the redshift surveys of the distribution of galaxies. The CMBR, last scattered at the epoch of decoupling, has to a high accuracy a black-body distribution [8], with a temperature $T_{0}=2.728 \pm 0.002 \mathrm{~K}$, which is almost independent of direction. The Differential Microwave Radiometer (DMR) experiment on the COBE satellite measured a tiny variation in intensity of the CMBR, at fixed frequency. This is equivalently expressed as a variation $\delta T$ in the temperature, which was measured to be $\delta T / T_{0} \approx 10^{-5}$ [9]. The four-year COBE data are fitted by a scale-free spectrum; the spectral index was found to be $n_{\mathrm{S}}$ $=1.2 \pm 0.3$ and the quadrupole anisotropy $Q_{\text {rms-PS }}$ $=15.3_{-2.8}^{+3.8} \mu \mathrm{K}$ [9]. The CMBR anisotropies spectrum is usually parametrized in terms of the multipole moments $C_{l}$, defined as the coefficients in the expansion of the temperature autocorrelation function

$$
\begin{aligned}
\left\langle\frac{\delta T}{T}\right. & \left.\left(\mathbf{e}_{1}\right) \frac{\delta T}{T}\left(\mathbf{e}_{2}\right)\right\rangle\left.\right|_{\left(\mathbf{e}_{1} \cdot \mathbf{e}_{2}=\cos \vartheta\right)} \\
& =\frac{1}{4 \pi} \sum_{l}(2 l+1) C_{l} P_{l}(\cos \vartheta),
\end{aligned}
$$

which compares points in the sky separated by an angle $\vartheta$. The value of $C_{l}$ is determined by fluctuations on angular scales of order $\pi / l$. The angular power spectrum of anisotropies observed today is usually given by the power per logarithmic interval in $l$, plotting $l(l+1) C_{l}$ versus $l$.

On large angular scales, the main physical mechanism which contributes to the redshift of photons propagating in a perturbed Friedmann geometry, originates from fluctuations in the gravitational potential on the last-scattering surface. The COBE-DMR experiment, which measured CMBR anisotropies on such large angular scales $(l \lesssim 20)$, confirmed the predicted scale-invariant spectrum and yields mainly a normalization for the different models of large-scale structure formation.

On intermediate angular scales, $0.1^{\circ} \leq \vartheta \leq 2^{\circ}$, the main contribution to the CMBR anisotropies comes from the intrinsic inhomogeneities on the surface of the last scattering, due to acoustic oscillations in the coupled baryon-radiation fluid prior to decoupling. On the same angular scales, there is a Doppler contribution to the CMBR anisotropies, due to the relative motions of emitter and observer. The sum of these two contributions is denoted by the term acoustic peaks. An analysis of recent CMBR flat-band measurements on inter- mediate angular scales gives [10] in the best-fit power spectrum a peak $\left[l(l+1) C_{l} / 2 \pi\right]^{1 / 2} T_{0}=76 \mu \mathrm{K}$ with $l=260$.

Among the various experiments measuring CMBR anisotropies, the Saskatoon experiment [11] is of particular importance since it relates [12] CMBR anisotropies to the power spectrum of matter density perturbations estimated through clustering properties of galaxies and clusters of galaxies. More precisely, the Saskatoon experiment measures temperature anisotropies for multipoles in the range $l \approx 80$ -400 , which corresponds to the range of wavelengths for which we have data on galaxy clusters.

Analyzing a large number of available data on redshifts of individual galaxies and Abell galaxy clusters, one obtains [12] the power spectrum for clusters of galaxies, over the wave-number interval from $k \approx 0.03 h \mathrm{Mpc}^{-1}$ to $k \approx 0.3$ $h \mathrm{Mpc}^{-1}$. On very large scales $\left(k<0.03 h \mathrm{Mpc}^{-1}\right)$, the large error bars are due to incomplete data. However, near the turn over, error bars are small, thus both the relative position and amplitude of the turn over are determined accurately. As discussed in, e.g. Ref. [12], the power spectrum reveals the existence of a nontrivial feature at a wave number $k_{0}=0.052 \pm 0.005 h \mathrm{Mpc}^{-1}$. Assuming this peak exists (further studies are necessary to confirm it), the amplitude of the observed power spectrum is larger near the peak by a factor 1.4 [12] with respect to the power spectrum of the standard cold dark matter model. The existence of this peak is not related [13] to acoustic oscillations in the tight coupled baryon-photon plasma. As stated in Ref. [13], the current CMBR experimental data combined with observational cluster data, favor theoretical models that have built-in a characteristic scale in their initial spectrum.

Recently, the COBE data have also been used to test the Gaussianity of the CMBR anisotropies. Three groups [1416] have now reported results showing that the fluctuations would not be Gaussian. The three groups work with different methods. In Ref. [14], the estimation of the bispectrum $B_{l}$ is used as a criterion to test Gaussianity. The dominant nonGaussian contribution has been found near $l=16$. It is clear that these results should be taken cautiously since, for example, the issue of foreground contamination could change the conclusions. However, the possibility of non-Gaussian statistics in the CMBR anisotropies should be taken into account seriously.

The Saskatoon measurements could be explained by playing with the values of the cosmological parameters. In particular, the value of the cosmological constant, $\Omega_{\Lambda} \approx 0.6$, recently inferred from the SNIa measurements could account for the high position of the first acoustic peak. But the other features (the presence of a peak in the power spectrum; nonGaussian fluctuations in the CMBR), if confirmed, clearly go beyond the paradigm of cold dark matter (CDM) and slow roll inflation. In order to explain them, different mechanisms have been advocated. For instance, double-inflation [17] or multiple-inflation [18] models have been used to explain the presence of the peak in the power spectrum. Another scenario can be offered within models where the inflaton field evolves through a kink in its potential [19]. To explain the non-Gaussianity, different mechanisms have been proposed. Of course, this appears naturally when the perturbations are 
induced by topological defects. However, even in the context of inflation, non-Gaussianity can be present, as, for example, in the case of stochastic inflation [20,21].

All these solutions are in fact different possible modifications of the power spectrum of the primordial fluctuations. In this article, our aim is to discuss the choice of the initial quantum state in which the quantum fields are placed. This choice is of course crucial for the determination of the primordial power spectrum, and different quantum states will lead to different power spectra. In the literature, it is (almost, see Ref. [22]) always assumed that the state of the perturbations is the vacuum (In a curved spacetime the definition of the vacuum state is not unique. A more precise definition of the vacuum used in this paper is given in what follows and coincides with the one in Ref. [23]):

$$
|0\rangle \equiv \underset{\mathbf{k}}{\otimes}\left|0_{\mathbf{k}}\right\rangle .
$$

Let us examine how this choice can be justified. Since this question is a problem of boundary conditions, it must be addressed by means of a theory of the initial conditions for the early Universe. Such a theory should rely on full quantum gravity, which is unknown at present. The only candidate at our disposal is quantum cosmology. Generally, it predicts that the initial state is indeed the vacuum. For example, the no-boundary choice for the wave function of the cosmological perturbations implies that the Bardeen operator is placed in the vacuum state, see Ref. [24]. This result does not come as a surprise since the Hartle-Hawking proposal is a generalization of a method that gives the ground-state wave function of a system in ordinary quantum mechanics.

However, although fascinating, quantum cosmology is not yet a well-developed branch of physics and many important questions remain unsolved to this day. To our knowledge, there exists no proof that quantum considerations automatically lead to a vacuum initial state for the perturbations. Such a proof, if it exists, should rely on full quantum gravity.

On the other hand, the choice of the vacuum is also based on the hypothesis that the initial state of the Universe should be a "maximally symmetric state" [25]. Concretely, this means that no scale should be privileged. This seems to be the simplest starting point. However, since the choice of the initial state is supposed to appear naturally in the context of quantum gravity, it could also be argued that such a privileged scale does exist and is equal to the Planck scale $l_{\mathrm{Pl}}$ $=\left(\hbar G / c^{3}\right)^{1 / 2} \approx 10^{-33} \mathrm{~cm}$. This becomes even more intriguing if one recalls that in order to solve the usual problems of the standard model of cosmology, one needs $60 e$ folds during inflation. This means that the Planck scale has now been stretched to a scale of at least $60 \mathrm{pc}$. Accordingly, all the wavelengths below $60 \mathrm{pc}$ were sub-Planckian at the moment of their generation. Of course, the structure of space-time below the Planck scale is unknown and it may be very different from the one we are used to. Probably, such notions as sub-Planckian wavelengths or even scale factor are meaningless in a regime where the gravitational quantum effects are important.
The arguments presented in the previous discussion show that it is worth studying nonvacuum initial states for cosmological perturbations. Rather than relying on theoretical arguments, our goal will be to allow for the possibility of nonvacuum initial states and to establish the consequences for the observables described at the beginning of the Introduction. We study whether choices other than the vacuum automatically lead to inconsistencies or conflicts with the available observations or if, on the contrary, there exists a window for the free parameters of the model, which fits the observational data.

Our choice of a nonvacuum initial state is guided by a very simple idea: the initial state could have a built-in characteristic scale since this seems to be the simplest way to generalize the vacuum state. The question now arises as for the physical origin of this scale. A possible answer is that the natural scale is the Planck length stretched by the cosmological expansion. It is clear that, so as not to be in conflict with observations, we would like this fundamental scale to be now translated to the characteristic scale $l_{\mathrm{C}} \approx 200 \mathrm{Mpc}$ $=6.2 \times 10^{26} \mathrm{~cm}$ (here, as in the rest of this article we take $h=0.5$ ). Since the ratio $l_{\mathrm{C}} / l_{\mathrm{Pl}}$ is given by $l_{\mathrm{C}} / l_{\mathrm{Pl}} \approx 10^{27} e^{N}$, where $N$ is the number of $e$-folds during inflation, this means that $N \approx 75$. Interestingly enough, we note that this leads to a number of $e$ folds greater than the minimum number required, i.e., 60. In the context of Linde's chaotic inflation, it is assumed that, initially, the inflaton potential $V(\varphi)$ is such that $V\left(\varphi_{i}\right) \approx m_{\mathrm{Pl}}^{4}$ where $m_{\mathrm{Pl}}$ is the Planck mass. If the potential is given by, e.g., $V(\varphi)=\left(\lambda / 4\right.$ !) $\varphi^{4}$, this leads to an initial value of the scalar field greater than $4.4 m_{\mathrm{Pl}}$, which is needed to get the usual $60 e$ folds. Consequently, this leads to a huge number of $e$ folds, $N \approx 10^{8}$. It is clear that, with such a number, the Planck length cannot be stretched to $200 \mathrm{Mpc}$ presently. Let us note that these models (with a large number of $e$ folds) suffer from the "super-Planck scale problem" [27]: all the scales of cosmological interest now were subPlanckian at the beginning of inflation. Since quantum field theory is expected to break down in this regime, the predictions of these models could be questionable. On the other hand, in the spirit of chaotic inflation itself, there exist regions of space in which the initial value of the field was $\varphi_{i}$ $\approx 4.9 m_{\mathrm{Pl}}$. This value leads to a number of $e$ folds equal to 75. Therefore, the model presented in this article is certainly more relevant in the case where inflation does not last for a long period. In chaotic inflation, the probability of having a long period of inflation is greater than the probability to get a small number of $e$ folds. Thus, our model does not fit very well within the chaotic inflation approach.

It should also be mentioned that it has been shown in Ref. [28] that a large class of initial states approaches the BunchDavis vacuum in the de Sitter spacetime. However, this class of initial states has a Gaussian wave functional and therefore the argument that the choice of a nonvacuum initial state would involve exponential fine tuning does not apply to the case considered here.

Recently, a model with a small number of $e$ folds has been constructed in Ref. [29]. This kind of models naturally arises in the context of supersymetric (SUSY) and supergravity (SUGRA) inflation. They are particulary well suited to 
the model put forward in this article. They consist in multiple bursts of inflation which in total last for $\approx 75$ expansion times. In addition, the last stage of inflation is preceded by other inflationary epochs. The "initial state" of this last epoch is the result of the evolution of the "true initial state" through the multiple preceding bursts of inflation. Clearly, there is no reason for assuming this "effective initial state" to be the vacuum. Let us emphasize that this argument holds for every model with many stages of inflation since, in this case, the origin of the characteristic scale could no longer be the Planck length stretched to $l_{\mathrm{C}}$ but could correspond to the time where one of the fields starts rolling down.

Our model possesses a privileged scale and therefore belongs to the class of models already envisaged in Ref. [26]. However, we would like to emphasize that the origin of this scale is physically completely different and we will point out that there exist observables, which, in principle, allow us to distinguish between the different models. A last comment on the fine tuning issue is in order here. It is true that the position of the characteristic scale must be chosen carefully. Otherwise the model would simply be in contradiction with the available data. We would like to emphasize that this is not a feature of our model only but in fact of all the BSI (broken scale invariant) models [26]. In this respect our model is similar to the other BSI models.

This paper is organized as follows. In Sec. II we discuss nonvacuum initial state for the cosmological perturbations. We first briefly describe the theory of perturbations of quantum-mechanical origin. We then describe the nonvacuum initial states considered in this article. We finally calculate the power spectra of the Bardeen potential for these states and show that it possesses either a step or a bump. In Sec. III we examine the observational consequences of the calculated power spectra; we compare our theoretical predictions with current experimental and observational data, which will fix the parameters of our model. We end with the conclusions given in Sec. IV.

\section{NONVACUUM INITIAL STATE FOR THE PERTURBATIONS}

\section{A. Perturbations of quantum-mechanical origin}

The background model is described by a FriedmannLemaitre-Robertson-Walker (FLRW) metric whose spacelike sections are flat $(c=1)$ : $\mathrm{d} s^{2}=a^{2}(\eta)\left(-\mathrm{d} \eta^{2}+\mathrm{d} \mathbf{x}^{2}\right)$. We assume that inflation is driven by a single scalar field, $\varphi_{0}(\eta)$. It is convenient to define the background quantities $\mathcal{H}(\eta)$ and $\gamma(\eta)$ by:

$$
\mathcal{H} \equiv a^{\prime} / a, \quad \gamma(\eta) \equiv 1-\frac{\mathcal{H}^{\prime}}{\mathcal{H}^{2}},
$$

where the primes denote the derivatives with respect to conformal time. In the case of the de Sitter space-time, $\gamma$ vanishes.

In the synchronous gauge, without loss of generality, the line element for the FLRW background plus scalar perturbations can be written as [30]:

$$
\begin{aligned}
\mathrm{d} s^{2}= & a^{2}(\eta)\left\{-\mathrm{d} \eta^{2}+\left[\delta_{i j}+\frac{1}{(2 \pi)^{3 / 2}} \int \mathrm{d} \mathbf{k}\right.\right. \\
& \left.\left.\times\left(h(\eta, \mathbf{k}) \delta_{i j}-\frac{h_{l}(\eta, \mathbf{k})}{k^{2}} k_{i} k_{j}\right) e^{i \mathbf{k} \cdot \mathbf{x}}\right] \mathrm{d} x^{i} \mathrm{~d} x^{j}\right\},
\end{aligned}
$$

where the functions $h, h_{l}$ represent the scalar perturbations of the gravitational field and the longitudinal-longitudinal perturbation, respectively. In the same manner, the perturbations of the scalar field are Fourier decomposed according to

$$
\delta \varphi(\eta, \mathbf{x})=\frac{1}{(2 \pi)^{3 / 2}} \int \mathrm{d} \mathbf{k} \varphi_{1}(\eta, \mathbf{k}) e^{i \mathbf{k} \cdot \mathbf{x}}
$$

The perturbed Einstein equations couple the scalar sector, $h$ and $h_{l}$, to the perturbed scalar field $\varphi_{1}$ : see Ref. [30]. It can be shown that the residual gauge invariant quantity $\mu(\eta, \mathbf{k})$ [30] defined by

$$
\mu \equiv \frac{a}{\mathcal{H} \sqrt{\gamma}}\left(h^{\prime}+\mathcal{H} \gamma h\right),
$$

can be used to express all other relevant quantities. The quantity $\mu(\eta, \mathbf{k})$ is related to the gauge-invariant Bardeen potential through the equation

$$
\Phi^{(\mathrm{SG})}=\frac{\mathcal{H} \gamma}{2 k^{2}}\left(\frac{\mu}{a \sqrt{\gamma}}\right)^{\prime},
$$

where "SG" means calculated in the synchronous gauge, see Ref. [31]. The quantity $\mu(\eta, \mathbf{k})$ is not defined in the de Sitter case. This case must be treated separately and for it we have $\Phi^{(\mathrm{SG})}=0$ : there are no density perturbations at all because the fluctuations of the scalar field are not coupled to the perturbations of the metric when the equation of state is $p=-\rho$. The perturbed Einstein equations imply that the equation of motion for $\mu(\eta, \mathbf{k})$ is given by [30]

$$
\mu^{\prime \prime}+\left[k^{2}-\frac{(a \sqrt{\gamma})^{\prime \prime}}{(a \sqrt{\gamma})}\right] \mu=0 .
$$

The above is the characteristic equation of a parametric oscillator whose time-dependent frequency depends on the scale factor and its derivative (up to $a^{(4)}$ ).

In this article, we assume that the perturbations are of quantum-mechanical origin. This hypothesis fixes completely the normalization of $\delta \varphi(\eta, \mathbf{x})$ and of the scalar perturbations. The normalization is fixed in the high-frequency regime. In this regime, the perturbed field can be considered as a free field in the curved FLRW background space-time. It is therefore necessary to study the quantization of such a free field denoted in the following by $\varphi(\eta, \mathbf{x})$. We first address this question and we then make the link between $\varphi(\eta, \mathbf{x})$ and $\delta \varphi(\eta, \mathbf{x})$. We choose to define the Fourier component of $\varphi(\eta, \mathbf{x})$ according to 


$$
\varphi(\eta, \mathbf{x})=\frac{1}{a(\eta)} \frac{1}{(2 \pi)^{3 / 2}} \int d \mathbf{k} \chi(\eta, \mathbf{k}) e^{i \mathbf{k} \cdot \mathbf{x}}
$$

where we have renormalized the time-dependent amplitude with the scale factor. The Fourier component satisfies $\chi(\eta$, $-\mathbf{k})=\chi^{*}(\eta, \mathbf{k})$, because the field is real. The action, given by

$$
\begin{aligned}
S & =\int d^{4} x \mathcal{L} \\
& =\frac{1}{2} \int d^{4} x a^{2}\left[\left[\varphi^{\prime}(\eta, \mathbf{x})\right]^{2}-\sum_{i}\left[\partial_{i} \varphi(\eta, \mathbf{x})\right]^{2}\right],
\end{aligned}
$$

can also be written in terms of the Fourier component $\chi(\eta, \mathbf{k})$. The result reads

$$
\begin{aligned}
S= & \int d \eta \int_{R^{3+}} d \mathbf{k} \overline{\mathcal{L}} \\
= & \int d \eta \int_{R^{3+}} d \mathbf{k}\left\{\left|\chi^{\prime}(\eta, \mathbf{k})\right|^{2}+\left(\frac{a^{\prime 2}}{a^{2}}-k^{2}\right)|\chi(\eta, \mathbf{k})|^{2}\right. \\
& \left.-\frac{a^{\prime}}{a}\left[\chi^{\prime}(\eta, \mathbf{k}) \chi^{*}(\eta, \mathbf{k})+\chi^{\prime *}(\eta, \mathbf{k}) \chi(\eta, \mathbf{k})\right]\right\} .
\end{aligned}
$$

The variation of the action leads to the equation of motion for the equation component $\chi^{\prime \prime}+\chi\left[k^{2}-a^{\prime \prime} / a\right]=0$. Again, we find a parametric oscillator-type equation. Of course, if there is no expansion, or if the Universe is in the radiationdominated era, it reduces to the usual equation of motion of an harmonic oscillator.

We now turn to the Hamiltonian formalism. The first step is the calculation of the momentum conjugate to $\varphi(\eta, \mathbf{x})$ defined by

$$
\pi(\eta, \mathbf{x}) \equiv \frac{\partial \mathcal{L}}{\partial\left[\varphi^{\prime}(\eta, \mathbf{x})\right]}=a^{2} \varphi^{\prime}(\eta, \mathbf{x})
$$

$\pi(\eta, \mathbf{x})$ can be expressed in terms of the momentum conjugate to $\chi(\eta, \mathbf{k})$,

$$
p(\eta, \mathbf{k}) \equiv \frac{\partial \overline{\mathcal{L}}}{\partial\left[\chi^{\prime *}(\eta, \mathbf{k})\right]}=\chi^{\prime}(\eta, \mathbf{k})-\frac{a^{\prime}}{a} \chi(\eta, \mathbf{k}),
$$

through the relation

$$
\pi(\eta, \mathbf{x})=\frac{a(\eta)}{(2 \pi)^{3 / 2}} \int d \mathbf{k} p(\eta, \mathbf{k}) e^{i \mathbf{k} \cdot \mathbf{x}}
$$

As a preparation to the quantization, the normal variable $\alpha(\eta, \mathbf{k})$ is introduced. Its definition is given by

$$
\alpha(\eta, \mathbf{k}) \equiv N(k)\left[\chi(\eta, \mathbf{k})+\frac{i}{k} p(\eta, \mathbf{k})\right],
$$

where $N(k)$ is, at the classical level, a free factor.

We are now in a position where quantization can be carried out. The field $\varphi(\eta, \mathbf{x})$ and its conjugate momentum $\pi(\eta, \mathbf{x})$ become operators that satisfy the commutation relation

$$
\left[\hat{\varphi}(\eta, \mathbf{x}), \hat{\pi}\left(\eta, \mathbf{x}^{\prime}\right)\right]=i \hbar \delta\left(\mathbf{x}-\mathbf{x}^{\prime}\right) .
$$

The normal variable $\alpha(\eta, \mathbf{k})$ becomes a dimensionless operator $c_{\mathbf{k}}(\eta)$ such that, at any time, $\left[c_{\mathbf{k}}(\eta), c_{\mathbf{k}^{\prime}}^{\dagger}(\eta)\right]=\delta_{\mathbf{k k}^{\prime}}$. With the help of Eqs. (9) and (16) and of the definition of the normal variable, Eq. (17), the field operator and the conjugate momentum operator can now be expressed in terms of the annihilation and creation operators $c_{\mathbf{k}}(\eta)$ and $c_{\mathbf{k}}^{\dagger}(\eta)$. The normalization factor $N(k)$ is fixed by the following argument: the energy of the scalar field is given by $E$ $=\int d^{3} x \sqrt{-g} \rho$ where $\rho=-T_{0}^{0}$ is the time-time component of the stress-energy tensor. Requiring that $E$ takes the following (usual) suggestive form in the high-frequency regime:

$$
E=\int d \mathbf{k} \frac{\hbar \omega}{2}\left[c_{\mathbf{k}} c_{\mathbf{k}}^{\dagger}+c_{\mathbf{k}}^{\dagger} c_{\mathbf{k}}\right]
$$

leads to

$$
N(k)=\sqrt{\frac{k}{2 \hbar}} .
$$

Therefore, the scalar field operator can be written as

$$
\hat{\varphi}(\eta, \mathbf{x})=\frac{\sqrt{\hbar}}{a(\eta)} \frac{1}{(2 \pi)^{3 / 2}} \int \frac{d \mathbf{k}}{\sqrt{2 k}}\left[c_{\mathbf{k}}(\eta) e^{i \mathbf{k} \cdot \mathbf{x}}+c_{\mathbf{k}}^{\dagger}(\eta) e^{-i \mathbf{k} \cdot \mathbf{x}}\right] .
$$

This equation no longer contains arbitrary (or unfixed) factors. The spirit of this argument is comparable to that of the method employed in Ref. [30].

The Hamiltonian can be deduced from the action in a straightforward manner and reads

$$
H=\frac{\hbar}{2} \int d \mathbf{k}\left[k\left(c_{\mathbf{k}} c_{\mathbf{k}}^{\dagger}+c_{-\mathbf{k}}^{\dagger} c_{-\mathbf{k}}\right)-i \frac{a^{\prime}}{a}\left(c_{\mathbf{k}} c_{-\mathbf{k}}-c_{\mathbf{k}}^{\dagger} c_{-\mathbf{k}}^{\dagger}\right)\right] .
$$

In the above expression, the first term represents the Hamiltonian of a set of harmonic oscillators, whereas the second term can be viewed as an interaction term between the perturbations and the background. This term is present only in a dynamical FLRW Universe since the coupling function is proportional to the first time derivative of the scale factor. The time evolution of the field operator is given by the time evolution of the creation and annihilation operators. It can be calculated using the Heisenberg equation

$$
i \hbar \frac{d}{d \eta} \hat{\varphi}(\eta, \mathbf{x})=[\hat{\varphi}(\eta, \mathbf{x}), H] .
$$


This equation can be solved by a Bogoliubov transformation, expressed as

$$
\begin{aligned}
& c_{\mathbf{k}}(\eta)=u_{k}(\eta) c_{\mathbf{k}}\left(\eta_{0}\right)+v_{k}(\eta) c_{-\mathbf{k}}^{\dagger}\left(\eta_{0}\right), \\
& c_{\mathbf{k}}^{\dagger}(\eta)=u_{k}^{*}(\eta) c_{\mathbf{k}}^{\dagger}\left(\eta_{0}\right)+v_{k}^{*}(\eta) c_{-\mathbf{k}}\left(\eta_{0}\right),
\end{aligned}
$$

where the functions $u_{k}(\eta)$ and $v_{k}(\eta)$ only depend on the norm of the vector $\mathbf{k}$. These functions are such that $\left|u_{k}\right|^{2}$ $-\left|v_{k}\right|^{2}=1$, so that the commutation relation between the creation and annihilation operators is preserved in time. The time $\eta_{0}$ must be thought of as the time where the initial conditions are fixed. Whatever these last ones are, we have $u_{k}\left(\eta_{0}\right)=1$ and $v_{k}\left(\eta_{0}\right)=0$. The differential equations that allow the determination of $u_{k}(\eta)$ and $v_{k}(\eta)$ are

$$
i u_{k}^{\prime}=k u_{k}+i \frac{a^{\prime}}{a} v_{k}^{*}, \quad i v_{k}^{\prime}=k v_{k}+i \frac{a^{\prime}}{a} u_{k}^{*} .
$$

If we introduce the Bogoliubov transformation given by Eqs. (24) and(25) in the expression of the field operator, Eq. (21), we obtain

$$
\begin{aligned}
\hat{\varphi}(\eta, \mathbf{x})= & \frac{\sqrt{\hbar}}{a(\eta)} \frac{1}{(2 \pi)^{3 / 2}} \int \frac{d \mathbf{k}}{\sqrt{2 k}} \\
& \times\left[c_{\mathbf{k}}\left(\eta_{0}\right)\left(u_{k}+v_{k}^{*}\right)(\eta) e^{i \mathbf{k} \cdot \mathbf{x}}\right. \\
& \left.+c_{\mathbf{k}}^{\dagger}\left(\eta_{0}\right)\left(u_{k}^{*}+v_{k}\right)(\eta) e^{-i \mathbf{k} \cdot \mathbf{x}}\right] .
\end{aligned}
$$

From Eq. (26), it is easy to see that the function ( $u_{k}$ $\left.+v_{k}^{*}\right)(\eta)$ satisfies the same equation as $\chi(\eta, \mathbf{k})$. In the highfrequency regime, the term $a^{\prime \prime} / a$ becomes negligible and we have $\lim _{k \rightarrow+\infty}\left(u_{k}+v_{k}^{*}\right)(\eta)=e^{-i k\left(\eta-\eta_{0}\right)}$. This means that, in this regime, the operator $\hat{\chi}(\eta, \mathbf{k})$ is given by

$$
\lim _{k \rightarrow+\infty} \hat{\chi}(\eta, \mathbf{k})=\sqrt{\hbar}\left[c_{\mathbf{k}}\left(\eta_{0}\right) \frac{e^{-i k\left(\eta-\eta_{0}\right)}}{\sqrt{2 k}}+c_{-\mathbf{k}}^{\dagger}\left(\eta_{0}\right) \frac{e^{i k\left(\eta-\eta_{0}\right)}}{\sqrt{2 k}}\right] .
$$

We can now come back to our initial problem, which consists in finding the correct normalization of the perturbed scalar field and of the scalar perturbations. We can identify the Fourier component operator of the perturbed field $\hat{\varphi}_{1}(\eta, \mathbf{k})$, with $\hat{\chi}(\eta, \mathbf{k}) / a(\eta)$, both operators being considered in the high-frequency regime. Let us emphasize again that this identification is valid only in this regime. Otherwise, the field $\hat{\varphi}_{1}(\eta, \mathbf{k})$ does not behave as the free field $\hat{\varphi}(\eta, \mathbf{x})$ and the time dependence of the modes is no longer given by the function $\left(u_{k}+v_{k}^{*}\right)(\eta)$. The normalization of the perturbed scalar field fixes automatically the normalization of the scalar perturbations of the metric since they are linked through Einstein's equations. We only need this link in the high-frequency regime. It can be expressed as (see Refs. $[30,31])$

$$
\lim _{k \rightarrow+\infty} \hat{\mu}(\eta, \mathbf{k})=-\sqrt{2 \kappa} a(\eta) \lim _{k \rightarrow+\infty} \hat{\varphi}_{1}(\eta, \mathbf{k}),
$$

where $\kappa \equiv 8 \pi G$. From the last expression and Eq. (28), we immediately deduce that

$$
\begin{aligned}
\lim _{k \rightarrow+\infty} & \hat{\mu}(\eta, \mathbf{k}) \\
& =-4 \sqrt{\pi} l_{\mathrm{P}[}\left[c_{\mathbf{k}}\left(\eta_{0}\right) \frac{e^{-i k\left(\eta-\eta_{0}\right)}}{\sqrt{2 k}}+c_{-\mathbf{k}}^{\dagger}\left(\eta_{0}\right) \frac{e^{i k\left(\eta-\eta_{0}\right)}}{\sqrt{2 k}}\right],
\end{aligned}
$$

where $l_{\mathrm{Pl}}=(G \hbar)^{1 / 2}$ is the Planck length. As announced, the normalization of the scalar perturbations is now completely determined. In general, the operator $\hat{\mu}(\eta, \mathbf{k})$ will be given by

$$
\hat{\mu}(\eta, \mathbf{k})=-4 \sqrt{\pi} l_{\mathrm{PI}}\left[c_{\mathbf{k}}\left(\eta_{0}\right) \xi_{k}(\eta)+c_{-\mathbf{k}}^{\dagger}\left(\eta_{0}\right) \xi_{k}^{*}(\eta)\right] .
$$

The function $\xi_{k}(\eta)$ is the solution of Eq. (8) such that $\lim _{k \rightarrow+\infty} \xi_{k}=e^{-i k\left(\eta-\eta_{0}\right)} / \sqrt{2 k}$. If we introduce the function $f_{k}(\eta)$ defined by

$$
f_{k}(\eta) \equiv-4 \sqrt{\pi}\left[(\mathcal{H} \gamma) /\left(2 k^{2}\right)\right]\left[\xi_{k} /(a \sqrt{\gamma})^{\prime}\right],
$$

then the dimensionless Bardeen operator $\hat{\Phi}(\eta, \mathbf{x})$ can be written as

$$
\begin{aligned}
\hat{\Phi}(\eta, \mathbf{x})= & \frac{l_{\mathrm{Pl}}}{(2 \pi)^{3 / 2}} \int d \mathbf{k}\left[c_{\mathbf{k}}\left(\eta_{0}\right) f_{k}(\eta) e^{i \mathbf{k} \cdot \mathbf{x}}\right. \\
& \left.+c_{\mathbf{k}}^{\dagger}\left(\eta_{0}\right) f_{k}^{*}(\eta) e^{-i \mathbf{k} \cdot \mathbf{x}}\right] .
\end{aligned}
$$

This equation is the main equation of this section and will be used in the following.

To conclude this part, it is interesting to consider the previous equations in the case of power law inflation, i.e., when the scale factor is given by

$$
a(\eta)=l_{0}|\eta|^{1+\beta} .
$$

To have inflation, $\beta$ must be such that $\beta+2<0$ (the case $-2<\beta<-1$ is not considered here because it cannot be realized with a single scalar field); $\beta=-2$ corresponds to the de Sitter universe; $l_{0}$ has dimension of length and, in the de Sitter case, it is equal to the Hubble radius $l_{H} \equiv a^{2} / a^{\prime}$. Moreover, in a de Sitter universe, the function $\gamma(\eta)$ turns out to be zero. We would like to notice that the assumption of power-law inflation is not as restrictive as it seems. Indeed, the widely used slow-roll approximation boils down to power-law inflation, with an effective $\beta$ depending on the slow-roll parameters, see Refs. [32]. In the case of power law inflation, analytical exact solutions for the equations of motion of the perturbations can be found. With the scale factor of Eq. (34), Eq. (8) can be solved in terms of Bessel functions. Then, the exact solution for the function $\xi_{k}(\eta)$ is

$$
\xi_{k}(\eta)=-i(\pi / 2)^{1 / 2} e^{i\left(k \eta_{0}-\pi \beta / 2\right)}(k \eta)^{1 / 2} H_{\beta+1 / 2}^{(2)}(k \eta) / \sqrt{2 k},
$$


where $H_{\beta+1 / 2}^{(2)}$ is the second-kind Hankel function of order $\beta+1 / 2$. It is straightforward to deduce the corresponding equation for $f_{k}(\eta)$ :

$$
f_{k}(\eta)=-\pi \sqrt{2} \frac{\mathcal{H} \sqrt{\gamma}}{k^{2}} e^{i\left(k \eta_{0}-\pi \beta / 2\right)} \frac{i k}{a} \frac{\sqrt{k \eta}}{\sqrt{2 k}} H_{\beta+3 / 2}^{(2)}(k \eta) .
$$

The previous expression for $f_{k}(\eta)$ guarantees that the field $\hat{\Phi}(\eta, \mathbf{x})$ possesses the correct behavior in the high-frequency regime. Roughly speaking,

$$
\lim _{k \rightarrow+\infty} f_{k}(\eta) \sim e^{-i k \eta} / \sqrt{2 k},
$$

with the correct amplitude. This should be the case of any field, regardless of the initial conditions one may choose.

\section{B. Quantum states}

The formulation of quantum field theory used in the previous subsection was written in the Heisenberg picture. So far, we have only calculated the time dependence of the Bardeen operator. In order to describe completely the system, one must in addition specify in which quantum state the field $\hat{\Phi}(\eta, \mathbf{x})$ is placed. As we already mentioned, it is usually found in the literature that the initial state of the perturbations is taken to be the vacuum. Here we address the hypothesis that the perturbations are initially in a nonvacuum state. Our choice of nonvacuum states is guided by the idea that one must introduce a scale in the theory. We denote the corresponding wave number by $k_{0}$. We examine three different nonvacuum states.

Let $\mathcal{D}$ be a domain in the momentum space characterized by the numbers $k_{0}$ and $\sigma$, such that $k_{0}$ is the privileged scale and $\sigma$ the dispersion around it. Concretely, we take $\mathcal{D}$ as the space between the spheres of radius $k_{0}-\sigma$ and $k_{0}+\sigma$, i.e., a shell of width $2 \sigma$ in $k$ space. The domain $\mathcal{D}$ is invariant under rotations and therefore is compatible with the assumption of isotropy of the Universe. Our first state is given by

$$
\begin{aligned}
\left|\Psi_{1}\left(k_{0}, \sigma, n\right)\right\rangle & \equiv \prod_{\mathbf{k} \in \mathcal{D}\left(k_{0}, \sigma\right)} \frac{\left(c_{\mathbf{k}}^{\dagger}\right)^{n}}{\sqrt{n !}}\left|0_{\mathbf{k}}\right\rangle \underset{\mathbf{p} \notin \mathcal{D}\left(k_{0}, \sigma\right)}{\otimes}\left|0_{\mathbf{p}}\right\rangle, \\
& =\underset{\mathbf{k} \in \mathcal{D}\left(k_{0}, \sigma\right)}{\otimes}\left|n_{\mathbf{k}}\right\rangle \underset{\mathbf{p} \notin \mathcal{D}\left(k_{0}, \sigma\right)}{\otimes}\left|0_{\mathbf{p}}\right\rangle .
\end{aligned}
$$

The state $\left|n_{\mathbf{k}}\right\rangle$ is an $n$-particle state satisfying, at $\eta=\eta_{0}$ : $c_{\mathbf{k}}\left|n_{\mathbf{k}}\right\rangle=\sqrt{n}\left|(n-1)_{\mathbf{k}}\right\rangle$ and $c_{\mathbf{k}}^{\dagger}\left|n_{\mathbf{k}}\right\rangle=\sqrt{n+1}\left|(n+1)_{\mathbf{k}}\right\rangle$.

More complicated states can be constructed by considering quantum superpositions of $\left|\Psi_{1}\left(k_{0}, \sigma, n\right)\right\rangle$. We will consider the following state:

$$
\left|\Psi_{2}\left(k_{0}, n\right)\right\rangle \equiv \int d \sigma g(\sigma)\left|\Psi_{1}\left(k_{0}, \sigma, n\right)\right\rangle,
$$

where, a priori, $g(\sigma)$ is an arbitrary function of $\sigma$. It is clear from the definition of the state $\left|\Psi_{1}\right\rangle$ that the transition be- tween the empty and the filled modes is sharp. Physically, this is probably not very realistic. The function $g(\sigma)$ will be chosen in order to "smooth out" the quantum state $\left|\Psi_{1}\right\rangle$. Also it should be clear that the writing of $\left|\Psi_{2}\right\rangle$ in Eq. (39) is symbolic. An accurate definition of this state requires to take into account subtleties, which will be considered when we calculate the spectrum in the next section.

Finally a third state can be defined according to

$$
\left|\Psi_{3}\left(k_{0}\right)\right\rangle \equiv \sum_{n=0}^{\infty} h(n)\left|\Psi_{2}\left(k_{0}, n\right)\right\rangle .
$$

The function $h(n)$ is arbitrary. As demonstrated below, this state will allow us to work with an effective number of quanta, which will no longer be an integer. This state seems to be the most natural rotational-invariant, smooth, quantum state that privileges a scale. Typically, the quantum state given in Eq. (40) depends on $k_{0}$ and on the free parameters characterizing the functions $g(\sigma)$ and $h(n)$. Their values will be determined later by confronting our theoretical predictions versus experimental and observational data. Our aim is now to calculate the power spectra of the Bardeen potential operator in the three states $\left|\Psi_{i}\right\rangle, \quad i=1,2,3$.

\section{Power spectra}

From now on, for convenience, we consider that the system is in a box whose edges have length $L$. As a consequence, the wave vector possesses discrete components given by $k_{i}=[(2 \pi) / L] m_{i}$, where $m_{i}$ is an integer. The Bardeen operator can be written as

$$
\begin{aligned}
\hat{\Phi}(\eta, \mathbf{x})= & \frac{l_{\mathrm{Pl}}}{L^{3 / 2}} \sum_{\mathbf{k}}\left[c_{\mathbf{k}}\left(\eta_{0}\right) f_{k}(\eta) e^{i \mathbf{k} \cdot \mathbf{x}}\right. \\
& \left.+c_{\mathbf{k}}^{\dagger}\left(\eta_{0}\right) f_{k}^{*}(\eta) e^{-i \mathbf{k} \cdot \mathbf{x}}\right] .
\end{aligned}
$$

We pass from the discrete representation to the continuous one by sending $L$ to infinity and by applying the rule $1 /(2 \pi)^{3} \int d \mathbf{k} \rightarrow 1 / L^{3} \Sigma_{\mathbf{k}}$. It is clear that the final result does not depend on $L$.

The power spectrum of $\hat{\Phi}(\eta, \mathbf{x})$ in the state $|\Psi\rangle$, denoted by $P_{\Phi}(k ;|\Psi\rangle)$, is defined through the calculation of the twopoint correlation function $K_{2}(r ;|\Psi\rangle)$. In the continuous limit,

$$
\begin{aligned}
K_{2}(r ;|\Psi\rangle) & \equiv \frac{\langle\Psi|\hat{\Phi}(\eta, \mathbf{x}) \hat{\Phi}(\eta, \mathbf{x}+\mathbf{r})| \Psi\rangle}{\langle\Psi \mid \Psi\rangle} \\
& =\int_{0}^{\infty} \frac{d k}{k} \frac{\sin k r}{k r} k^{3} P_{\Phi}(k ;|\Psi\rangle) .
\end{aligned}
$$

In this definition, we have taken into account the fact that the state $|\Psi\rangle$ is not automatically normalized to 1 . The power spectrum is a time-dependent function but in the longwavelength limit this dependence disappears. In order to perform the computation of the correlation function for the state $\left|\Psi_{1}\right\rangle$, one needs the following quantities: 


$$
\begin{gathered}
\left\langle\Psi_{1}\left(k_{0}, \sigma, n\right)\left|c_{\mathbf{p}} c_{\mathbf{q}}\right| \Psi_{1}\left(k_{0}, \sigma, n\right)\right\rangle \\
=\left\langle\Psi_{1}\left(k_{0}, \sigma, n\right)\left|c_{\mathbf{p}}^{\dagger} c_{\mathbf{q}}^{\dagger}\right| \Psi_{1}\left(k_{0}, \sigma, n\right)\right\rangle=0, \\
\left\langle\Psi_{1}\left(k_{0}, \sigma, n\right)\left|c_{\mathbf{p}} c_{\mathbf{q}}^{\dagger}\right| \Psi_{1}\left(k_{0}, \sigma, n\right)\right\rangle=n \delta(\mathbf{q} \in \mathcal{D}) \delta_{\mathbf{p q}}+\delta_{\mathbf{p q}}, \\
\left\langle\Psi_{1}\left(k_{0}, \sigma, n\right)\left|c_{\mathbf{p}}^{\dagger} c_{\mathbf{q}}\right| \Psi_{1}\left(k_{0}, \sigma, n\right)\right\rangle=n \delta(\mathbf{q} \in \mathcal{D}) \delta_{\mathbf{p q}} .
\end{gathered}
$$

In these formulas, $\delta(\mathbf{q} \in \mathcal{D})$ is a function that is equal to 1 if $\mathbf{q} \in \mathcal{D}$ and 0 otherwise.

As a warm up, we calculate the power spectrum for the state $\left|\Psi_{1}\right\rangle$ with $n=0$, i.e., for the vacuum. Using the previous equations in the definition of the correlation function, Eq. (42), one finds

$$
K_{2}(r ;|0\rangle)=\frac{l_{\mathrm{Pl}}^{2}}{L^{3}} \sum_{\mathbf{k}}\left|f_{k}\right|^{2} e^{-i \mathbf{k} \cdot \mathbf{r}},
$$

which in the continuous limit $L \rightarrow+\infty$ goes to

$$
\frac{l_{\mathrm{Pl}}^{2}}{(2 \pi)^{3}} \int d^{3} \vec{k}\left|f_{k}\right|^{2} e^{-i \mathbf{k} \cdot \mathbf{r}} \text {. }
$$

After having performed the angular integrations, we recover the power spectrum $P_{\Phi}(k ;|0\rangle)$ :

$$
k^{3} P_{\Phi}(k ;|0\rangle)=\frac{l_{\mathrm{Pl}}^{2}}{2 \pi^{2}} k^{3}\left|f_{k}\right|^{2} .
$$

Let us turn to the calculation of $K_{2}\left(r ;\left|\Psi_{1}\right\rangle\right.$. It can be expressed as

$$
K_{2}\left(r ;\left|\Psi_{1}\right\rangle\right)=\frac{l_{\mathrm{Pl}}^{2}}{L^{3}} \sum_{\mathbf{k}}\left(\left|f_{k}\right|^{2} e^{-i \mathbf{k} \cdot \mathbf{r}}[1+2 n \delta(\mathbf{k} \in \mathcal{D})]\right) .
$$

From this equation and from the definition of the function $\delta(\mathbf{k} \in \mathcal{D})$, we deduce the expression of the power spectrum

$$
\begin{aligned}
k^{3} P_{\Phi}\left(k ;\left|\Psi_{1}\right\rangle\right)= & \frac{l_{\mathrm{Pl}}^{2}}{2 \pi^{2}} k^{3}\left|f_{k}\right|^{2}\left\{1+2 n\left[\mathrm { H } \left(k-k_{0}+\sigma\right.\right.\right. \\
& \left.\left.-\mathrm{H}\left(k-k_{0}-\sigma\right)\right]\right\},
\end{aligned}
$$

where $H$ is a Heaviside function. We see that, in addition to the usual vacuum spectrum, there is a new contribution located around the wave number $k_{0}$. This new contribution vanishes if $n=0$, as expected.

This spectrum is not continuous. As already mentioned, this is not physically very realistic. It has for origin the very crude definition of the state $\left|\Psi_{1}\right\rangle$. We thus turn to the case where the quantum state is given by $\left|\Psi_{2}\right\rangle$. This refinement will allow us to obtain a smooth and physical spectrum.

Since the system is placed in a box, the state $\left|\Psi_{2}\right\rangle$ can be defined by a discrete sum according to

$$
\left|\Psi_{2}\left(k_{0}, n\right)\right\rangle \equiv \sum_{i=0}^{N} g_{i}\left|\Psi_{1}\left(k_{0}, \sigma_{i}, n\right)\right\rangle
$$

where $g_{i}$ and $\sigma_{i}, i=0, \ldots, N$, are just series of numbers. We choose the $\sigma_{i}$ 's such that

$$
\left\langle\Psi_{1}\left(k_{0}, \sigma_{i}, n\right) \mid \Psi_{1}\left(k_{0}, \sigma_{j}, n\right)\right\rangle=\delta_{i j}
$$

This is satisfied if the number of modes in the domains $\mathcal{D}\left(k_{0}, \sigma_{i}\right)$ and $\mathcal{D}\left(k_{0}, \sigma_{j}\right), \mathcal{N}\left(\mathcal{D}_{i}\right)$ and $\mathcal{N}\left(\mathcal{D}_{j}\right)$, respectively, are such that $\mathcal{N}\left(\mathcal{D}_{i}\right)-\mathcal{N}\left(\mathcal{D}_{j}\right) \geqslant 1$. This condition boils down to $\sigma_{i}-\sigma_{j} \geqslant \pi^{2} /\left[L^{3}\left(k_{0}^{2}+\sigma_{i}^{2}\right)\right]$, where we have assumed that $\sigma_{i}-\sigma_{j} \ll 1$. Therefore, we can always find a value of $L$ such that the condition be fulfilled. Then, the calculation of $\left\langle\Psi_{2}|\hat{\Phi}(\eta, \mathbf{x}) \hat{\Phi}(\eta, \mathbf{x}+\mathbf{r})| \Psi_{2}\right\rangle$ can be performed. The result reads

$$
\begin{aligned}
\left\langle\Psi_{2}|\hat{\Phi}(\eta, \mathbf{x}) \hat{\Phi}(\eta, \mathbf{x}+\mathbf{r})| \Psi_{2}\right\rangle & \\
= & \frac{l_{\mathrm{P} 1}^{2}}{L^{3}} \sum_{\mathbf{k}}\left|f_{k}\right|^{2} e^{-i \mathbf{k} \cdot \mathbf{r}}\left\{\left[\sum_{i=0}^{N}\left|g_{i}\right|^{2}\right]\right. \\
+ & \left.2 n\left[\sum_{i=0}^{N}\left|g_{i}\right|^{2} \delta\left(\mathbf{k} \in \mathcal{D}\left(k_{0}, \sigma_{i}\right)\right)\right]\right\} .
\end{aligned}
$$

Our aim is to calculate $G(k) \equiv \sum_{i=0}^{N}\left|g_{i}\right|^{2} \delta\left(\mathbf{k} \in \mathcal{D}_{i}\right)$ [for convenience $\mathcal{D}\left(k_{0}, \sigma_{i}\right)$ is denoted by $\left.\mathcal{D}_{i}\right]$. By symmetry, $G\left(k_{0}\right.$ $\left.-k^{\prime}\right)=G\left(k_{0}+k^{\prime}\right)$, so that we will consider the case $k \equiv k_{0}$ $+k^{\prime}, k^{\prime} \geqslant 0$. In this sum $k^{\prime}$ is fixed. As a consequence, there exists an integer $i_{0}$ such that if $i<i_{0}, \delta\left(\mathbf{k} \in \mathcal{D}_{i}\right)=0$ and if $i \geqslant i_{0}$, then $\delta\left(\mathbf{k} \in \mathcal{D}_{i}\right)=1$, or, equivalenty, $\sigma_{i_{0}}<k^{\prime}$ $\leqslant \sigma_{i_{0}+1}$. This means that the sum $\sum_{i=0}^{N}\left|g_{i}\right|^{2} \delta\left(\mathbf{k} \in \mathcal{D}_{i}\right)$ is in fact equal to $\sum_{i=i_{0}}{ }^{N}\left|g_{i}\right|^{2}$. We choose the $\sigma_{i}$ 's and the coefficients $g_{i}$ according to

$$
\sigma_{i} \equiv i \frac{X_{\max }}{N}, \quad\left|g_{i}\right|^{2} \equiv-\frac{X_{\max }}{N} \times\left.\frac{d F}{d x}\right|_{x=\sigma_{i}},
$$

where $F$ is any decreasing function such that $F\left(X_{\max }\right)=0$. Then we have

$$
\sum_{i=0}^{N}\left|g_{i}\right|^{2} \delta\left(\mathbf{k} \in \mathcal{D}_{i}\right)=-\frac{X_{\max }}{N} \sum_{i=i_{0}}^{N} F^{\prime}\left(i \frac{X_{\max }}{N}\right)
$$

The last step is to send $N$ to infinity. This means that we consider a continuous series of intervals $\mathcal{D}_{i}$. We obtain

$$
\lim _{N \rightarrow+\infty} \sum_{i=0}^{N}\left|g_{i}\right|^{2} \delta\left(\mathbf{k} \in \mathcal{D}_{i}\right)=-\int_{k^{\prime}}^{X_{\max }} F^{\prime}(x) d x=F\left(k^{\prime}\right),
$$

by definition of the Riemann integral. In the same manner, $\sum_{i=0}^{N}\left|g_{i}\right|^{2}=F(0)$. In what follows, we take

$$
G(k)=F\left(k^{\prime}\right) \equiv e^{-\left(k-k_{0}\right)^{2} / \Sigma^{2}},
$$


where $\Sigma$ is a free parameter. This function does not exactly satisfy the assumptions made previously, but it is easy to show that the final result is free of these limitations and is in fact valid for any function $F$. It is clear that other functions are possible, but only the approximate shape of the distribution is important and the Gaussian is the prototype of the function we have in mind. The spectrum is obtained after having introduced the previous result in Eq. (53) and having taken the limit $L \rightarrow+\infty$. We obtain

$$
k^{3} P_{\Phi}\left(k ;\left|\Psi_{2}\right\rangle\right)=\frac{l_{\mathrm{Pl}}^{2}}{2 \pi^{2}} k^{3}\left|f_{k}\right|^{2}\left(1+2 n e^{-\left(k-k_{0}\right)^{2} / \Sigma^{2}}\right) .
$$

In this equation, it is clear that $n$ is an integer. We now show that this condition can be relaxed if the system is placed in the state $\left|\Psi_{3}\right\rangle$.

To calculate the spectrum for this state, it is sufficient to notice that $\left\langle\Psi_{2}\left(k_{0}, n\right) \mid \Psi_{2}\left(k_{0}, m\right)\right\rangle=\delta_{m n}$. Using this formula, straightforward calculations lead to

$$
k^{3} P_{\Phi}\left(k ;\left|\Psi_{3}\right\rangle\right)=\frac{l_{\mathrm{Pl}}^{2}}{2 \pi^{2}} k^{3}\left|f_{k}\right|^{2}\left(1+2 n_{\mathrm{eff}} e^{-\left(k-k_{0}\right)^{2} / \Sigma^{2}}\right),
$$

where the effective number of quanta $n_{\text {eff }}$ is given by

$$
n_{\mathrm{eff}}=\frac{\sum_{n=0}^{\infty} n|h(n)|^{2}}{\sum_{n=0}^{\infty}|h(n)|^{2}} .
$$

An attractive choice for the function $h(n)$ is obviously $h(n) \equiv e^{-\beta n}$ [this $\beta$ has of course nothing to do with the $\beta$ defined in Eq. (34)]. In this case, $n_{\text {eff }}$ is given by

$$
n_{\mathrm{eff}}=\frac{e^{-2 \beta}}{1-e^{-2 \beta}} .
$$

The spectra of Eqs. (58) and (59) are the main results of this section. Clearly, they possess a peak around the scale $k_{0}$. The position of the peak is controlled by the value of $k_{0}$, its width by $\Sigma$ and its height by $n$ or $n_{\text {eff }}$ (in fact by $\beta$ in the last case).

We will need the primordial spectrum only for large wavelengths. In the case of power law inflation, everything can be calculated exactly. In this limit, we have

$$
k^{3} P_{\Phi}(k ;|0\rangle)=A_{\mathrm{S}} k^{n_{\mathrm{S}}-1},
$$

with

$$
A_{\mathrm{S}}=\frac{l_{\mathrm{Pl}}^{2}}{l_{0}^{2}} \frac{\gamma(1+\beta)^{2}}{2^{2 \beta+4} \cos ^{2}(\beta \pi) \Gamma^{2}(\beta+5 / 2)}, \quad n_{\mathrm{S}}=2 \beta+5 .
$$

The above expression is strictly speaking not applicable in the case of a de Sitter universe, since then there are no scalar metric perturbations and the function $\gamma(\eta)$ turns out to be zero. The generation of density perturbations is only possible after the transition from the exponential inflationary era to the radiation-dominated Universe. If during inflation the Universe was very close to the de Sitter space-time, then the spectrum of density perturbations today is the so-called Harrison-Zel'dovich spectrum $\left(n_{\mathrm{S}}=1\right)$. All expressions derived in this section are still valid for $\beta \leqq-2$. The initial power spectrum in the case where the Bardeen operator is placed in the state $\left|\Psi_{2}\right\rangle$ can be written as

$$
k^{3} P_{\Phi}\left(k ;\left|\Psi_{2}\right\rangle\right)=A_{\mathrm{S}} k^{n_{\mathrm{S}}-1}\left(1+2 n e^{-\left(k-k_{0}\right)^{2} / \Sigma^{2}}\right) .
$$

If the state is $\left|\Psi_{3}\right\rangle$, we just have to replace the integer $n$ with the real number $n_{\text {eff }}$. Let us note that if, instead of considering intervals of the form $\left[k_{0}-\sigma, k_{0}+\sigma\right]$, one considers intervals such as $\left.] 0, k_{0}+\sigma\right]$ or $\left[k_{0}-\sigma, \infty[\right.$, which still privilege a scale, it is possible to build steplike spectra, the step being located at the scale $k_{0}$.

Recently in the literature, BSI spectra have also been studied, see Ref. [26]. In these articles, the privileged scale arises as a privileged energy in the inflaton potential (more precisely, a discontinuity, or a rapid variation, in the inflaton potential if first derivatives are present). We would like to stress that, in our case, the different physical origin of the privileged scale would in principle allow us to distinguish the different models. Indeed, in Ref. [26], the fluctuations are Gaussian. In the case studied here, the three-point correlation function still vanishes

$$
\left\langle\frac{\delta T}{T}\left(\mathbf{e}_{1}\right) \frac{\delta T}{T}\left(\mathbf{e}_{2}\right) \frac{\delta T}{T}\left(\mathbf{e}_{3}\right)\right\rangle=0,
$$

but the four-point correlation function no longer satisfies the relation

$$
\left\langle\left(\frac{\delta T}{T}(\mathbf{e})\right)^{4}\right\rangle=3\left[\left\langle\left(\frac{\delta T}{T}(\mathbf{e})\right)^{2}\right\rangle\right]^{2},
$$

which is typical of Gaussian statistics. The reason for this is clear. The ground-state wave function of an harmonic oscillator is a Gaussian and, as a consequence, the CMBR correlation functions for the vacuum exhibit Gaussian properties. On the other hand, the wave function of a state with a nonvanishing number of quanta is no longer a Gaussian and, correspondingly, the correlation functions deviate from Gaussianity. Therefore, a measure of the four-point correlation function (as well as any higher-order even-point correlation function) would permit to distinguish between the class of models presented here and the models of Ref. [26]. If it turns out that the type of non-Gaussianity apparently detected recently [14-16] is really present in the CMBR map, then these two classes of BSI models (as well as standard inflation) are ruled out, because they both predict a vanishing three-point correlation function. But if it turns out that some non-Gaussianity is present in the CMBR at the level of the four-point correlation function then the models presented here could account for this. 


\section{COMPARISON WITH OBSERVATIONS}

The aim of this section is to confront the power spectra given by Eq. (64) with observations. We will not use any accurate statistical methods to find the best values of the free parameters $k_{0}, \Sigma$, and $n / n_{\text {eff }}$, because we just want to obtain crude constraints. For this purpose we will use observations of the CMBR anisotropies and of the matter power spectrum.

We choose to work with the following cosmological parameters: the Hubble parameter is $h=0.5$, the baryonic matter-density parameter is $\Omega_{b}=0.05$, the density parameter $\Omega_{0} \equiv \Omega_{c}+\Omega_{b}+\Omega_{\Lambda}$ is equal to $1\left(\Omega_{c}\right.$ and $\Omega_{\Lambda}$ are, respectively, the CDM and the $\Lambda$-density parameters), there is no significant reionization and the spectral index is $n_{\mathrm{S}}=1$, when there is no contribution from the gravitational waves. Let us emphasize again that in this case, since it corresponds to a de Sitter phase, the Eq. (63) giving the normalization of the spectrum is strictly speaking not applicable. However, all expressions derived in the previous section can be applied for $n_{\mathrm{S}} \lesssim 1$. We will later discuss the case of small deviations from a scale-invariant (Harrison-Zel'dovich) spectrum, including the contribution of gravitational waves in the CMBR anisotropies. We will consider two different values for the cosmological constant density parameter $\Omega_{\Lambda} \equiv \Lambda /\left(3 H_{0}^{2}\right)$ and the sum of baryon-matter density parameter and CDM density parameter $\Omega_{m} \equiv \Omega_{b}+\Omega_{c}$, that is $\Omega_{\Lambda}=0, \Omega_{c}=0.95$ (hereafter denoted SCDM), and $\Omega_{\Lambda}=0.6, \Omega_{c}=0.35$ (hereafter denoted $\Lambda$ CDM). We point out that we have not assumed any biasing in the galaxy distribution with respect to the underlying mass fluctuations (the bias parameter is equal to 1$)$.

The spectrum must be normalized, i.e., the value of $A_{\mathrm{S}}$ must be determined. For this purpose, we use the value of $Q_{\text {rms-PS }}=T_{0}\left(5 C_{2} / 4 \pi\right)^{1 / 2} \sim 18 \mu \mathrm{K}\left(T_{0}=2.7 \mathrm{~K}\right)$ measured by the COBE satellite. We use the value $Q_{\text {rms-PS }} \sim 18 \mu \mathrm{K}$ because we have assumed that the spectrum is scale invariant. In the large-wavelength approximation, we have $\delta T / T$ $\sim(1 / 3) \Phi$. In addition the transfer function for the Bardeen potential can be taken equal to 1 (with an appropriate normalization). As a consequence the multipole can be written as

$$
\begin{aligned}
C_{l}= & \frac{4 \pi}{9} \int_{0}^{+\infty} \frac{d k}{k}\left[j_{l}\left[k\left(\eta_{0}-\eta_{\mathrm{LSS}}\right)\right]^{2} A_{\mathrm{S}}\left(n_{\mathrm{S}}\right) k^{n_{\mathrm{S}}-1}\right. \\
& \times\left(1+2 n e^{\left.\left.-\left(k-k_{0}\right)^{2} / \Sigma^{2}\right)\right]},\right.
\end{aligned}
$$

where $j_{l}$ is a spherical Bessel function of order $l$, and $\eta_{0}$ and $\eta_{\text {LSS }}$ denote, respectively, the conformal times now and at the last scattering surface. Let us remark that the $A_{\mathrm{S}}$ in the last expression is not exactly the $A_{\mathrm{S}}$ in Eqs. (62) and (63). Since the difference is not important for our purpose, we have kept the same notation. The previous expression can be evaluated explicitly. For the quadrupole, the result reads

$$
\begin{aligned}
C_{2}= & \frac{4 \pi}{9} A_{\mathrm{S}}\left(n_{\mathrm{S}}\right)\left(\eta_{0}-\eta_{\mathrm{LSS}}\right)^{1-n_{\mathrm{S}}} \\
& \times\left(\frac{\pi}{2^{4-n_{\mathrm{S}}}} \frac{\Gamma\left[3-n_{\mathrm{S}}\right] \Gamma\left[2+\left(n_{\mathrm{S}}-1\right) / 2\right]}{\Gamma^{2}\left[\left(4-n_{\mathrm{S}}\right) / 2\right] \Gamma\left[4-\left(n_{\mathrm{S}}-1\right) / 2\right]}+2 n I\right),
\end{aligned}
$$

where

$$
I \equiv \int_{0}^{+\infty} \frac{d u}{u^{2-n_{s}}}\left[j_{2}(u)\right]^{2} e^{-\left(u-u_{0}\right)^{2} / U^{2}},
$$

with $u_{0} \equiv k_{0}\left(\eta_{0}-\eta_{\text {LSS }}\right)$ and $U \equiv \Sigma\left(\eta_{0}-\eta_{\text {LSS }}\right)$. In what follows, we take $\eta_{0}-\eta_{L S S}=1$. The integral will be evaluated numerically for different values of the free parameters. We just have to specialize the last equation to a scale-invariant spectrum to obtain the following value for $A_{\mathrm{S}}$ :

$$
A_{\mathrm{S}}=\frac{108}{5 T_{0}^{2}} Q_{\mathrm{rms}-\mathrm{PS}}^{2} \frac{1}{1+24 n I}=\frac{9.4 \times 10^{-10}}{1+24 n I}
$$

In terms of the band power $\delta T_{l}$ defined by $\delta T_{l} \equiv T_{0}[l(l$ $\left.+1) C_{l} / 2 \pi\right]^{1 / 2}$, we find $\delta T_{2}=\sqrt{12 / 5} Q_{\text {rms-PS }}=27.9 \mu \mathrm{K}$.

We must choose the three parameters $k_{0}, n$, and $\Sigma$. Recently, it has been emphasized by many authors [12] that the power spectrum seems to contain large amplitude features at the scale $l_{\mathrm{C}} \approx 100 h^{-1} \mathrm{Mpc}$, which corresponds to a wave number equal to $0.062 \mathrm{~h} \mathrm{Mpc}^{-1}$. No other value for a privileged scale has been detected so far, and therefore any other choice would either lie in an unobservable range, or be in conflict with the data available at present. Consequently, we choose

$$
k_{0}=0.062 h \mathrm{Mpc}^{-1}=0.031 \mathrm{Mpc}^{-1} \text {, }
$$

with our value of the Hubble constant. Let us turn to the choice of the variance $\Sigma$. We have seen that the simplest nonvacuum initial states can lead to a power spectrum with either a bump or a step. In this article, we will restrict ourselves to the study of the bump case. Steplike spectra have already been studied in Ref. [26] and our conclusions would be similar. Therefore we will consider (rather arbitrarily, but the conclusion does not depend on the exact value of $\Sigma$, as long as it is not too large):

$$
\Sigma=0.3 k_{0}=0.0186 h \mathrm{Mpc}^{-1}
$$

From now on, we will always take these two values for $k_{0}$ and $\Sigma$ in any of the plots shown. In Fig. 1 we display the initial power spectrum for a few values of $n_{\text {eff }}$. The difference between $n_{\text {eff }}=0$ and $n_{\text {eff }} \neq 0$ is obvious.

In the case considered here, the integral $I$ is equal to $I\left(\Sigma=0.3 k_{0}\right) \approx 1.3 \times 10^{-6}$. It is completely negligible and will be taken equal to zero. This arises from the fact that the quadrupole is mainly fed by very large wavelengths (of the order of today's Hubble radius), whereas the bump occurs at much smaller wavelengths (of the order of the Hubble radius at the time of decoupling). Thus, the calculation of the quadrupole, and therefore the normalization, is not modified by the presence of the bump.

Let us discuss the matter power spectrum. The power spectrum can either be obtained by the Boltzmann code developed by one of us (A.R.) or by means of analytical fits. In this case, the baryon power spectrum is given by 


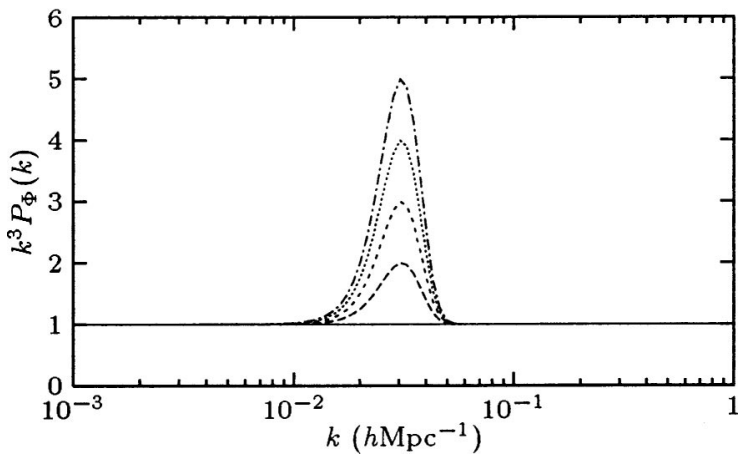

FIG. 1. Initial power spectrum for $n_{\text {eff }}$ ranging from 0 to 2 with steps of 0.5 . Vertical units are arbitrary.

$$
\frac{\delta \rho_{\mathrm{b}}}{\rho_{\mathrm{b}}} \equiv|\delta(k)|^{2}=A T^{2}(k) \frac{g^{2}\left(\Omega_{0}\right)}{g^{2}\left(\Omega_{m}\right)} k\left[1+2 n e^{-\left(k-k_{0}\right)^{2} / \Sigma^{2}}\right]
$$

where the different terms in this equation are explained below; $T(k)$ is the transfer function, which can be approximated by the following numerical fit [33]:

$$
\begin{aligned}
T(k)= & \frac{\ln (1+2.34 q)}{2.34 q} \\
& \times\left[1+3.89 q+(16.1 q)^{2}+(5.46 q)^{3}+(6.71 q)^{4}\right]^{-1 / 4},
\end{aligned}
$$

with $q \equiv k /\left[(h \Gamma) \mathrm{Mpc}^{-1}\right]$ where $\Gamma$ is the so-called shape parameter, which can be written as [34]

$$
\Gamma \equiv \Omega_{m} h e^{-\Omega_{b}-\Omega_{b} / \Omega_{m}} .
$$

The function $g(\Omega)$ takes into account the modification induced in the power spectrum by the presence of a cosmological constant. Its expression can be written as [35]

$$
g(\Omega) \equiv \frac{5 \Omega}{2}\left[\Omega^{4 / 7}-\Omega_{\Lambda}+\left(1+\frac{\Omega}{2}\right)\left(1+\frac{\Omega_{\Lambda}}{70}\right)\right]^{-1} .
$$

Finally the coefficient $A$ is the normalization. We normalize the spectrum to $\mathrm{COBE}$ data. This leads to the following value for $A$ :

$$
\begin{aligned}
A & =\left(2 l_{H}\right)^{4} \frac{6 \pi^{2}}{5} \frac{Q_{\mathrm{rms}-\mathrm{PS}}^{2}}{T_{0}^{2}} \frac{1}{1+24 n I} \\
& =\frac{6.82 \times 10^{5}}{1+24 n I} h^{-4} \mathrm{Mpc}^{4},
\end{aligned}
$$

where the Hubble radius $l_{H}$ is equal to $3000 h^{-1} \mathrm{Mpc}$.

We plot the multipole moments and the power spectrum for different values of $n$ and/or $n_{\text {eff }}$. The $C_{l}$ 's are obtained from the Boltzmann code previously used for the power spectrum. In all figures for the $C_{l}$ 's, we represent the COBE data [36] by diamonds, the Saskatoon data [37] by squares, and the CAT [38] data by crosses. (For clarity we have not displayed all CMBR data in the figures.) In all figures for

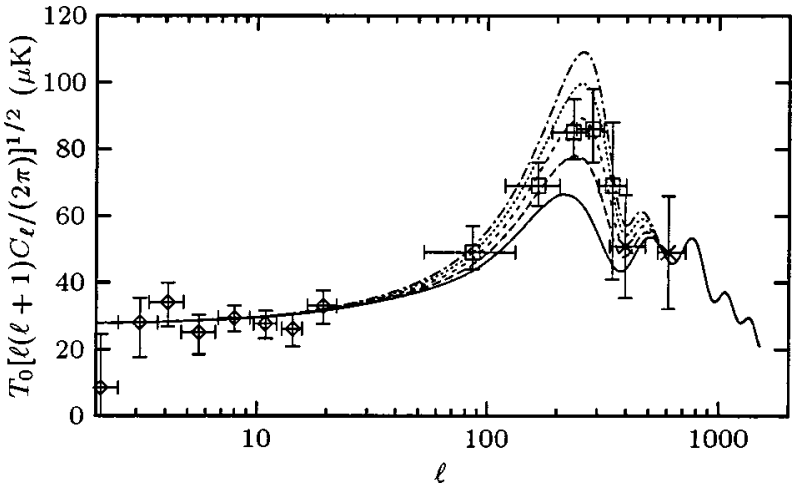

FIG. 2. Multipole moments for the SCDM model with $n_{\text {eff }}$ (and $n$ if it is integer) ranging from 0 to 2 with steps of 0.5 (from the bottom to the top). Diamonds represent COBE data, squares the Saskatoon data, and crosses the CAT data.

the power spectra, we represent the APM data [39] by diamonds, the velocities field measurements [40] by squares, and the data given by Einasto et al. [12] by crosses.

\section{A. Scalar modes only}

We first display the CMBR anisotropies in the scalar cold dark matter (SCDM) model (Fig. 2). In the case were $\Lambda$ $=0$, Saskatoon data are compatible with the case $n_{\text {eff }}=1$ (third curve).

We note that the position of the first Doppler peak is no longer around $l \approx 220$. Usually, its position is determined by the angular size of the Hubble radius at recombination. In our case, we must superimpose the bump present in the initial spectrum, the position of which is not at $l \approx 220$ but rather at the angular scale sustained by the built-in scale. As a consequence, the resulting peak is shifted towards higher values of $l$ for the values of the parameters considered here $(l \approx 260$ ). In addition, it could be difficult to distinguish the effect due to the primordial bump from the one coming from a variation of the cosmological parameters, thus increasing the degeneracy among the free parameters of the model. Let us note, however, that the bump in the initial power spectrum, should be easier to detect in the matter power spectrum since it is a more slowly varying function, as shown in Fig. 3.

A higher value of $n_{\text {eff }}$ ( 2 rather than 1) seems to be needed to explain the data of Einasto et al., but different cosmological parameters might lead to a better agreement between CMBR and matter power spectrum data. We now display the CMBR (Fig. 4) and matter power spectrum (Fig. 5) of the cold dark matter with a cosmological constant ( $\Lambda \mathrm{CDM})$ model.

When the cosmological constant $\Omega_{\Lambda}=0.6$, the early integrated Sachs-Wolfe effect already boosts the $l \simeq 200-300$ scale sufficiently [41]: at $n_{\text {eff }}=1$ this effect already puts too much power on these scales. A different value for $k_{0}$ and $\Sigma$ might also be needed to remain compatible with the CAT data. For the matter power spectrum, the same conclusion as for the SCDM model holds, that is a higher value of $n_{\mathrm{eff}}$ is preferred (around 2 or 3 ). 


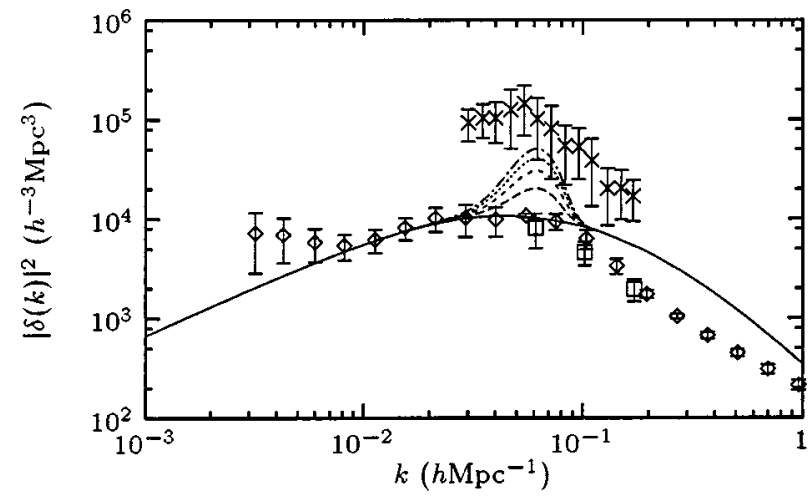

FIG. 3. Power spectrum for the SCDM model, with $n_{\text {eff }}$ ranging from 0 to 2 with step of 0.5 (from the bottom to the top). Diamonds represent the APM data, squares the velocities field measurements, and crosses the data by Einasto et al.

As a conclusion of this rapid analysis, we stress that our model is much more constrained if one imposes $n_{\text {eff }}$ to be an integer instead of a real number. Moreover, our model tends to favor a moderate value of $n_{\text {eff }}$ as well as a low value of the cosmological constant if the data of Einasto et al. are confirmed, or a low value of $n_{\text {eff }}$ and a high value of the cosmological constant (i.e., the currently popular cosmological model, with vacuum initial state) in the other case. It is easy to notice from Eq. (60), that since $n_{\text {eff }}$ is quite small, $h(n)$ is peaked around small values of $n$, and therefore the allowed window for the effective number of quanta is constrained to be around small values. In conclusion, the initial state found is not too far from the vacuum.

\section{B. Scalar and tensor modes}

One should also consider the contribution of the gravitational waves in the CMBR anisotropies. The data currently available are in fact the sum of the scalar plus the tensor contributions to the CMBR anisotropies. We recall that since there are two modes of polarization for the CMBR photons and that one of them is only generated by gravitational waves, it is in principle possible to distinguish between the scalar and tensor contributions to the CMBR anisotropies, see Ref. [42]. In what follows, we consider some standard inflationary predictions for gravitational waves: we take $n_{\mathrm{S}}$

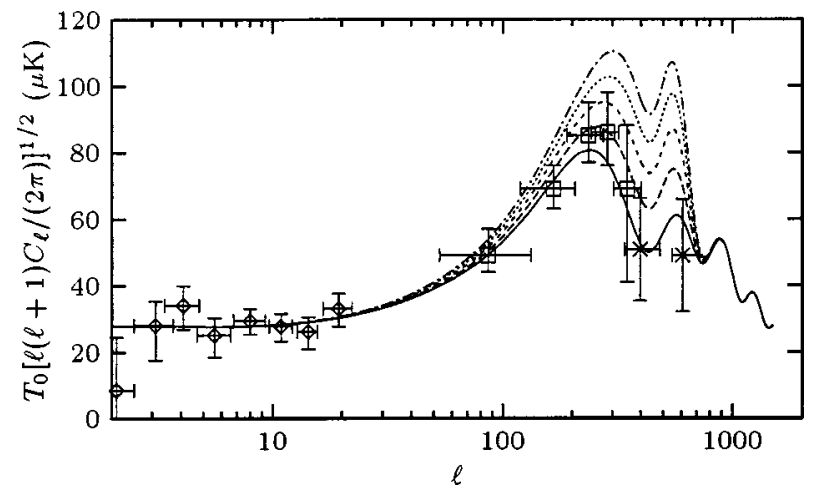

FIG. 4. Same as Fig. 2, but for the $\Lambda \mathrm{CDM}$ model, with $\Omega_{\Lambda}$ $=0.6$.

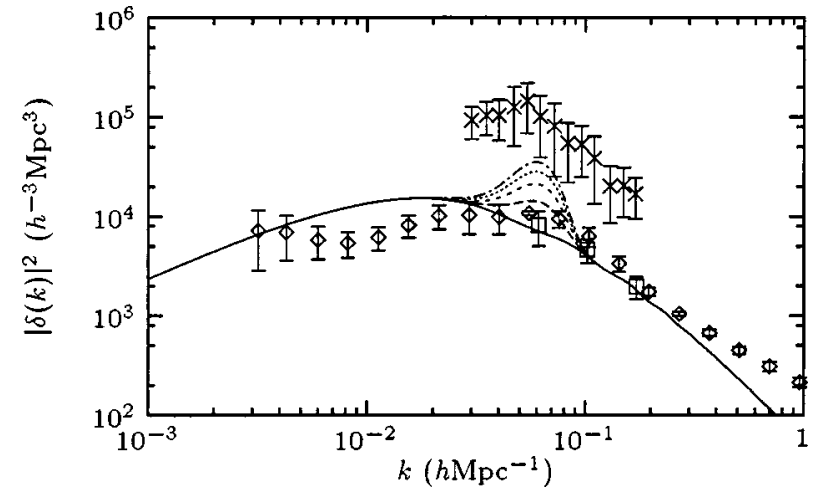

FIG. 5. Same as Fig. 3, but for the $\Lambda \mathrm{CDM}$ model.

$=0.9, n_{\mathrm{T}}=n_{\mathrm{S}}-1 \approx-0.1$ (the last equation being rigorous in the case of power-law inflation only), and the ratio of scalar to tensor amplitude $C_{2}^{\mathrm{T}} / C_{2}^{\mathrm{S}} \approx-7 n_{\mathrm{T}}$. In Fig. 6, we decompose CMBR anisotropies, showing the contributions from scalar and tensor modes separately.

In any model, the gravitational waves contribution can be important only for multipoles $l \lesssim 100$, while it is negligible at smaller angular scales (roughly speaking, the gravitational waves contribution is two orders of magnitude smaller at $l$ $\approx 300$ than at the quadrupole). The effect of gravitational waves is therefore to boost power on large angular scales (or, equivalently, to lower the height of the acoustic peaks with respect to the height of the low $l$ plateau). The fact that one observes an excess of power on small angular scales (with Saskatoon data), favors a low contribution from gravitational waves (which is in agreement with most inflationary models). In our model, the possibility to have a bump in the initial power spectrum enables us to boost the height of the acoustic peaks, and therefore to have some non-negligible contribution from gravitational waves: normalizing at COBE data, one imposes the value of $A_{\mathrm{S}}+A_{\mathrm{T}}$ instead of $A_{\mathrm{S}}$. As a result, the scalar perturbations amplitude $A_{\mathrm{S}}$ is smaller. Since the first acoustic peak depends only on scalar perturbations, we must keep the same value as before for the product $A_{\mathrm{S}}\left(1+2 n \exp \left[-\left(k_{0}-k_{\text {peak }}\right)^{2} / \Sigma^{2}\right]\right)$, which permits a higher value of $n_{\text {eff }}$ ( $k_{\text {peak }}$ is the characteristic wave number of the first Doppler peak).

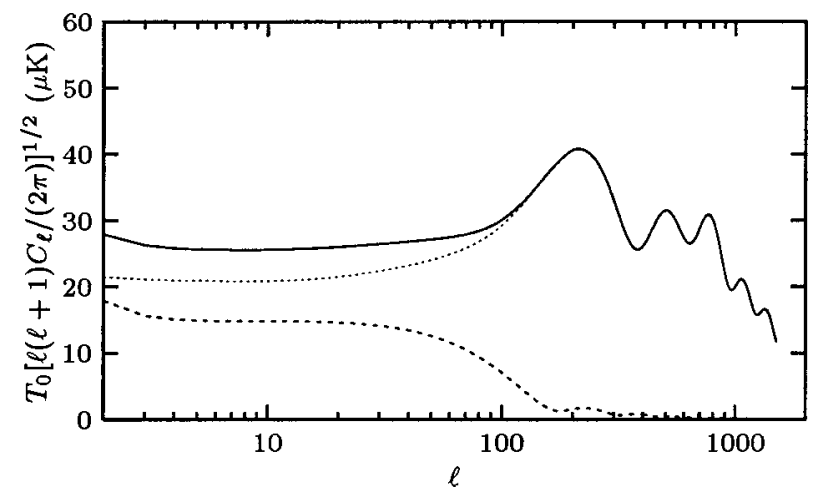

FIG. 6. CMBR anisotropies decomposition, showing scalar (dotted line) and tensor (dashed line) contributions. The total contribution is given by the solid line. 


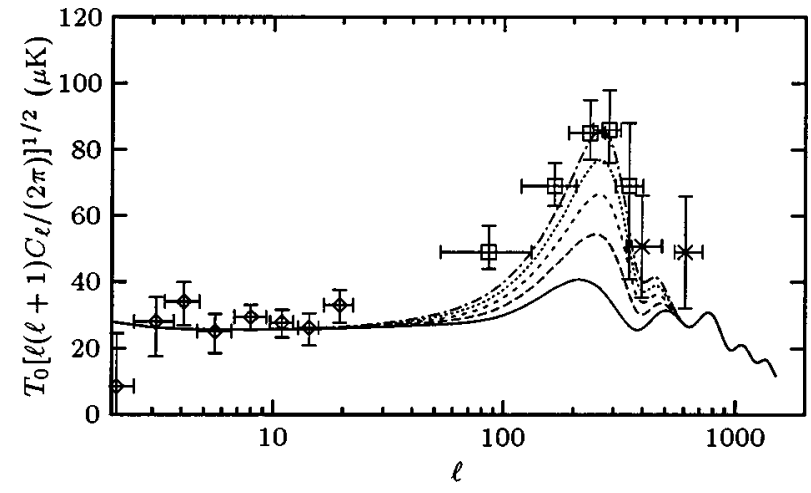

FIG. 7. CMBR anisotropies for the SCDM model, with $n_{\text {eff }}$ ranging from 0 to 4 with a step of 1 (from the bottom to the top). Both scalar and tensor contributions are included. Diamonds represent COBE data, squares the Saskatoon data, and crosses the CAT data.

In Figs. 7 and 8 we show the CMBR anisotropies and the matter power spectrum for the SCDM model, including both scalar and tensor contributions. Comparing these figures with Figs. 2 and 3, it can be concluded that if both scalar and tensor modes are included in the calculation of the multipole moments $C_{l}$ 's, then a higher number of quanta $(\simeq 4)$ is required as expected.

Finally, in Figs. 9 and 10 we show the CMBR anisotropies and the matter power spectrum for the $\Lambda$ CDM model including both scalar and tensor contributions. The same conclusions as for the SCDM model hold, but we note again that, as for the case without gravitational waves, matter power spectrum data favor a higher value of $n_{\text {eff }}$ than CMBR anisotropies data. We emphasize that when the gravitational waves contribution is not negligible, the standard case $n_{\text {eff }}$ $=0$ is excluded, and that extra power in the initial state is necessary.

\section{CONCLUSIONS}

In this paper we address the question of whether nonvacuum initial states for cosmological perturbations are allowed, or whether they are ruled out on the basis of present

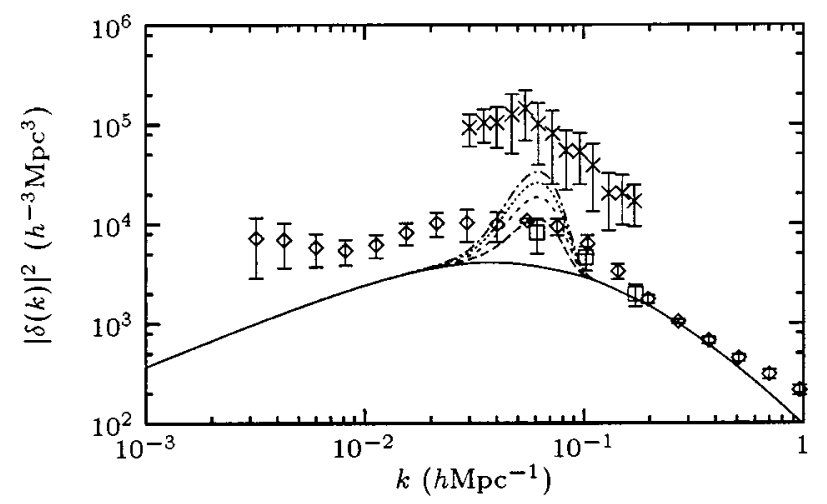

FIG. 8. Power spectrum for the SCDM model, with $n_{\text {eff }}$ ranging from 0 to 4 with steps of 1 . Diamonds represent the APM data, squares the velocities field measurements, and crosses the data given by Einasto et al.

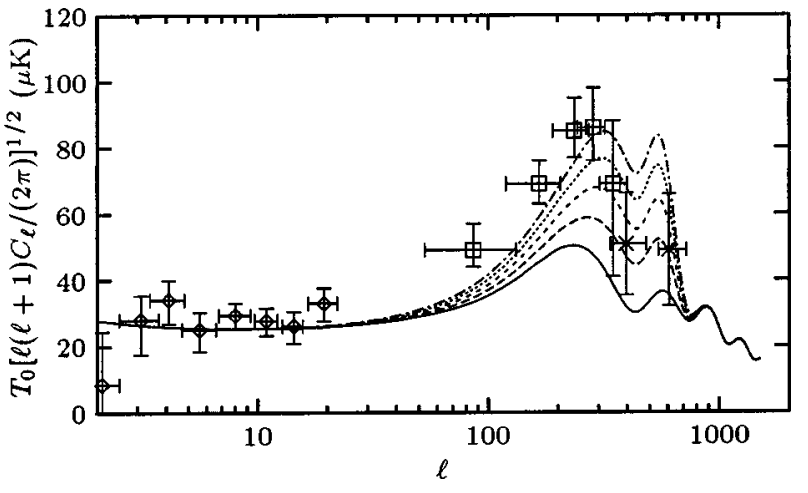

FIG. 9. Same as Fig. 7, but for the $\Lambda$ CDM model and with $n_{\text {eff }}$ ranging from 0 to 4 with a step of 1 (from the bottom to the top).

experimental and observational data.

The choice of the initial quantum state in which the quantum fields are placed should be made on the basis of full quantum gravity. Since this theory is at present unknown, we believe, as we discussed in the Introduction, that it is worth studying nonvacuum initial states for cosmological perturbations. Our choice of a nonvacuum initial state is guided by the idea that the initial state could have a built-in characteristic scale. We examined three different nonvacuum states, which are compatible with the assumption of isotropy of the Universe. Of particular interest is our choice of state $\left|\Psi_{3}\right\rangle$, which seems to be the most natural rotational-invariant smooth quantum state, which privileges a scale. We calculated the power spectra of the Bardeen potential for these three states and compared their theoretical predictions with current experimental and observational data, namely the CMBR anisotropy measurements and the redshift surveys of the distribution of galaxies. With our choice of initial states, the power spectra of the Bardeen potential possess a peak, around the wave number, that corresponds to the built-in characteristic scale of our model. The height of the peak is controlled by the number of quanta $n$ of the initial state and its width by another free parameter of our model. If the initial state is a quantum superposition then the height of the peak is controlled by the number $n_{\text {eff }}$, which does not need to be an integer.

The angular power spectrum of CMBR anisotropies for a

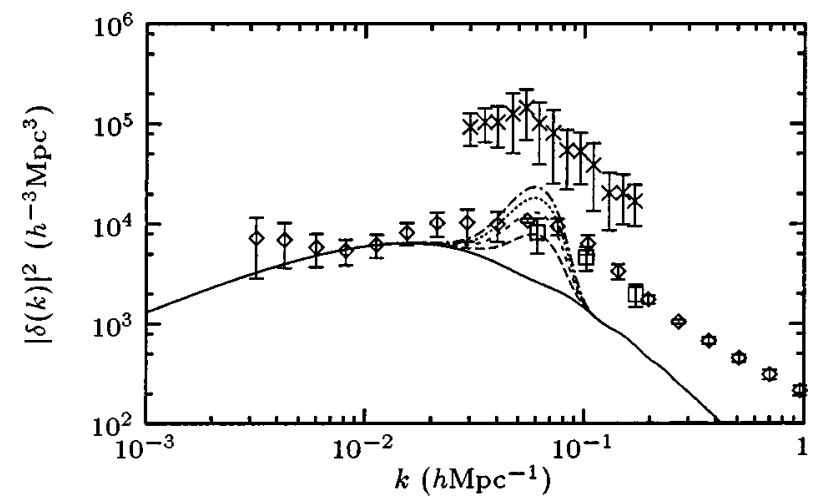

FIG. 10. Same as Fig. 8, but for the $\Lambda \mathrm{CDM}$ model and with $n_{\text {eff }}$ ranging from 0 to 4 with a step of 1 (from the bottom to the top). 
model with vanishing cosmological constant, tells us that the characteristics of the first acoustic peak, as revealed by the Saskatoon experiment, are compatible with the case $n_{\text {eff }}=1$. In the presence of a cosmological constant, CMBR anisotropy measurements are in agreement with $n_{\text {eff }}=0$ or $n_{\text {eff }}$ $=1$, depending on the value of the cosmological parameters. The observational data for the matter power spectra, as given by Einasto et al., favor higher values of the number of $n_{\mathrm{eff}}(2$ or 3), whatever the value of the cosmological constant.

The most realistic case is the one for which the sum of scalar and tensor modes contributions is included. Considering standard inflationary predictions for gravitational waves, we find that CMBR anisotropies measurements require a higher value of $n_{\text {eff }}$ ( 3 or 4) for both types of models, with and without a cosmological constant, than in the case of an absence of tensor modes contribution. This is in agreement with the matter power spectra. The analysis of the redshift surveys by Einasto et al. leads to matter power spectra that favor higher values of $n_{\text {eff }}$, once tensor contributions are also included. The interpretation of these results for the states $\left|\Psi_{2}\right\rangle$ and $\left|\Psi_{3}\right\rangle$ leads to the conclusion that since $n$ and $n_{\text {eff }}$ cannot be higher than a few, these states must be close to the vacuum.
In conclusion, if the initial state of the cosmological perturbations is not the vacuum but, instead, has a built-in characteristic scale, then generic predictions of the model are a high amplitude of the first acoustic peak, a nontrivial feature in the matter power spectrum, and deviations from Gaussianity in the CMBR map. It is too early to say whether the results of the Saskatoon experiment (see also Ref. [43]), as well as the analysis performed recently by Einasto et al., are first steps in this direction. More data are needed and future experiments will be important in determining whether the class of models proposed here provides an explanation which allows a better description of the observations than the standard paradigm of slow roll inflation plus cold dark matter.

\section{ACKNOWLEDGMENTS}

It is a pleasure to thank Robert Brandenberger for useful exchanges of comments. Discussions with Nathalie Deruelle, Ruth Durrer, Alejandro Gangui, and David Langlois are also acknowledged. We would also like to thank Volker Müller, who provided us with the cluster data.
[1] A. H. Guth and S.-Y. Pi, Phys. Rev. Lett. 49, 1110 (1982); S. W. Hawking, Phys. Lett. 115B, 295 (1982); A. A. Starobinsky ibid. 117B, 175 (1982).

[2] T. W. B. Kibble, Phys. Rep. 67, 183 (1980).

[3] R. Durrer, A. Gangui, and M. Sakellariadou, Phys. Rev. Lett. 76, 579 (1996).

[4] R. Durrer and M. Sakellariadou, Phys. Rev. D 56, 4480 (1997).

[5] U-L. Pen, U. Seljak, and N. Turok, Phys. Rev. Lett. 79, 1611 (1997).

[6] A. Albrecht, R. A. Battye, and J. Robinson, Phys. Rev. D 59, 023508 (1999).

[7] L. P. Grishchuk, Class. Quantum Grav. 10, 2449 (1993).

[8] J. C. Mather et al., Astrophys. J. Lett. 354, L37 (1990); E. S. Cheng et al., ibid. 456, L71 (1996).

[9] G. F. Smoot et al., Astrophys. J. Lett. 396, L1 (1992); C. L. Bennett et al., ibid. 464, L1 (1996).

[10] C. H. Lineweaver and D. Barbosa, Astrophys. J. 496, 624 (1998).

[11] C. B. Netterfield et al., Astrophys. J. 474, 47 (1997); E. Wollack et al., ibid. 476, 440 (1997).

[12] J. Einasto et al., Nature (London) 385, 139 (1997).

[13] F. Atrio-Barandela et al., J. Exp. Theor. Phys. 66, 397 (1998).

[14] P. G. Ferreira, J. Magueijo, and K. M. Gorski, Astrophys. J. Lett. 503, L1-L4 (1998); J. Magueijo, P. Ferreira, and K. Gorski, "Evidence for non-Gaussianity in the CMB,", astro-ph/9810414.

[15] J. Pando, D. Valls-Gabaud, and L. Fang, Phys. Rev. Lett. 81, 4568 (1998).

[16] D. Novikov, H. Feldman, and S. Shandarin, Int. J. Mod. Phys. D 8, 291 (1999).

[17] L. A. Kofman et al., Phys. Lett. 157B, 361 (1985); L. A. Kof- man and A. D. Linde, Nucl. Phys. B282, 555 (1987); L. A. Kofman and D. Yu. Pogosyan, Phys. Lett. B 214, 1753 (1988); S. Gottlöber et al., Phys. Rev. D 43, 2510 (1991); D. Polarski and A. A. Starobinsky, Nucl. Phys. B385, 623 (1992).

[18] J. A. Adams et al., Phys. Lett. B 391, 271 (1997); Nucl. Phys. B503, 405 (1997); M. Sakellariadou and N. Tetradis, "Spectrum of Cosmological Perturbations from Multiple-Stage Inflation,'” hep-ph/9806461.

[19] A. A. Starobinsky, Pis'ma Zh. Eksp. Teor. Fiz. 55, 477 (1992) [JETP Lett. 55, 489 (1992)].

[20] A. Gangui, Phys. Rev. D 50, 3684 (1994); A. Gangui, F. Lucchin, S. Mattarese, and S. Mollerach, Astrophys. J. 430, 447 (1994).

[21] A. Matacz, Phys. Rev. D 55, 1860 (1997).

[22] J. Lesgourges, D. Polarski, and A. A. Starobinski, Nucl. Phys. B497, 479 (1997).

[23] R. Brandenberger, Nucl. Phys. B245, 328 (1984).

[24] J. J. Halliwell and S. W. Hawking, Phys. Rev. D 31, 1777 (1985); J. J. Halliwell (unpublished).

[25] A. A. Starobsinky, Pis'ma Zh. Eksp. Teor. Fiz. 30, 719 (1979) [JETP Lett. 30, 682 (1979)].

[26] J. Lesgourgues, D. Polarski, and A. A. Starobinsky, Mon. Not. R. Astron. Soc. 297, 769 (1998); 308, 281 (1999); J. Lesgourgues, S. Prunet, and D. Polarski, ibid. 303, 45 (1999).

[27] R. H. Brandenberger (private communication).

[28] R. H. Brandenberger and C. T. Hill, Phys. Lett. B 179, 30 (1986).

[29] J. A. Adams, G. G. Ross, and S. Sarkar, Nucl. Phys. B503, 405 (1997).

[30] L. P. Grishchuk, Phys. Rev. D 50, 7154 (1994).

[31] J. Martin and D. J. Schwarz, Phys. Rev. D 57, 3302 (1998).

[32] J. E. Lidsey et al., Rev. Mod. Phys. 69, 373 (1997). 
[33] J. Bardeen et al., Astrophys. J. 304, 15 (1986).

[34] N. Sugiyama, Astrophys. J., Suppl. 100, 281 (1995).

[35] C. S. Smith et al., "Linearizing the Observed Power Spectrum," astro-ph/9702099, v2.

[36] G. Hinshaw et al., Astrophys. J. Lett. 464, L17 (1996).

[37] C. B. Netterfield, Astrophys. J. 474, 47 (1997).

[38] P. F. S. Scott et al., Astrophys. J. Lett. 461, L1 (1996); J. Baker, in "Proceedings of Particle Physics and the Early Universe Conference,' Cambridge, 1997, URL http:// www.mrao.cam.ac.uk/ppeuc/astronomy/paper/baker/ baker.html

[39] C. M. Baugh and G. Efstathiou, Mon. Not. R. Astron. Soc.
265, 145 (1993); E. Gaztañaga and C. M. Baugh, “Testing deprojection algorithms on mock angular catalogues: Evidence for a break in the power spectrum," astro-ph/9704246.

[40] T. Kolatt and A. Dekel, astro-ph/9512132.

[41] W. Hu, in Proceedings of the Universe at High-z, Large Scale Structure and the Cosmic Microwave Background, edited by E. Martinez-Gonzalez and J. L. Sanz (Springer Verlag, Berlin, 1996), p. 207.

[42] W. Hu and M. White, Phys. Rev. D 56, 596 (1997).

[43] K. Coble et al., "Anisotropy in the Cosmic Microwave Background at Degree Angular Scales: Python V Results," astro-ph/9902195. 